\title{
Dificultades en el aprendizaje del álgebra, un estudio con pruebas estandarizadas
}

\author{
Difficulties in learning algebra, a study with standardized tests
}

\author{
Luis Armando Ramos Palacios* \\ ORCID iD 0000-0002-8532-9862 \\ Marvin Iván Guifarro** \\ ORCID iD 0000-0003-4223-2187 \\ Luis Manuel Casas García*** \\ ORCID iD 0000-0002-2752-1784
}

\begin{abstract}
Resumen
El presente trabajo es un estudio orientado a identificar las principales dificultades que muestran los estudiantes al resolver problemas algorítmicos o algebraicos. Se ha tomado como referencia la base de datos que contiene la información sobre la aplicación de pruebas estandarizadas de matemáticas, a escala nacional, de estudiantes de $7^{\circ}$ a $9^{\circ}$ grado (doce a quince años) de la educación básica en Honduras. Después de un análisis de los parámetros estadísticos de dificultad, correlación, así como del análisis gráfico de las opciones de cada ítem, se seleccionaron los ítems contestados de forma incorrecta por estudiantes que tienen altos puntajes en las pruebas aplicadas, lo que permitió analizarlos tomando como referencia investigaciones realizadas en otros contextos. Los resultados muestran que estudiantes de alto desempeño cometen errores en problemas básicos de aritmética y álgebra. Consideramos que reconocer estos errores es un buen camino para orientar procesos de formación y capacitación docente, así como para fortalecer estrategias didácticas encaminadas a evitar los errores identificados.
\end{abstract}

Palabras clave: Dificultades. Errores. Matemáticas. Evaluación. Álgebra.

\begin{abstract}
This work is a study aimed at identifying the main difficulties that students show when solving algorithmic or algebraic problems. As a reference point, we used the database that contains the results of the application of standardized mathematics tests on basic education students from 7th to 9th grade (ages 12 to 15) in Honduras. After an analysis of the statistical parameters of difficulty, correlation, as well as the graphical analysis of the options of each item, the items answered incorrectly by students who had high scores in the applied tests were selected, which allowed them to be analyzed taking as reference the research carried out in other contexts. The results show that high performance students make mistakes in basic problems of arithmetic and algebra. We believe that recognizing these errors is a good way to guide teacher education and training processes, as well as to strengthen teaching strategies aimed at avoiding the identified errors.
\end{abstract}

Keywords: Difficulties. Mistakes. Mathematics. Evaluation. Algebra

\footnotetext{
* Doctor en Educación por la Universidad de Extremadura, España (UEx). Profesor Titular del Departamento de Matemáticas de la Universidad Pedagógica Nacional Francisco Morazán (UPNFM), Tegucigalpa, Honduras. Email: luramosp@upnfm.edu.hn.

** Máster en Matemática Educativa por la Universidad Pedagógica Nacional Francisco Morazán (UPNFM), Tegucigalpa, Honduras E-mail: marvguifarro@gmail.com.

*** Doctor en Psicopedagogía por la Universidad de Extremadura, España (UEx). Profesor Titular de Universidad en el área de Didáctica de las Matemáticas en la Universidad de Extremadura, Badajoz, España. E-mail: luisma@unex.es.
} 


\section{Introducción}

La enseñanza de las matemáticas en la educación básica de Honduras ( $1^{\circ}$ a $9^{\circ}$ grado) está fundamentada en el Currículo Nacional Básico (CNB), y para su implementación y seguimiento de una manera ordenada se han establecido estándares educativos nacionales; estos estándares, de acuerdo con el documento oficial, definen con claridad lo que un estudiante debe saber y saber hacer al finalizar un grado escolar (SECRETARÍA DE EDUCACIÓN, 2011).

Para valorar el logro de estos estándares, desde el año 2007, con el apoyo de expertos internacionales en evaluación educativa se ha venido realizando evaluaciones anuales, mediante la aplicación de pruebas escritas a estudiantes de los nueve grados de la educación básica nacional. Estas pruebas, llamadas también pruebas estandarizadas, han sido diseñadas, aplicadas y analizadas utilizando técnicas psicométricas, que aseguran la confiabilidad y la validez de sus resultados (SECRETARÍA DE EDUCACIÓN, 2017, 2018).

Las pruebas estandarizadas de matemáticas constan de preguntas de opción múltiple, con cuatro alternativas cada una, y son aplicadas a muestras nacionales de estudiantes de centros educativos públicos y privados del país. En algunos años, la aplicación ha sido a nivel censal.

Para evaluar el área de matemáticas en los grados de $7^{\circ}, 8^{\circ}$ y $9^{\circ}$, se incluyen preguntas orientadas a conocer el nivel de dominio de aspectos algorítmicos relacionados con operaciones aritméticas, así como los contenidos relacionados con álgebra básica, estos bloques tienen el mayor porcentaje de cobertura en la enseñanza de las matemáticas en estos grados (RAMOS; CASAS, 2018).

El diseño y construcción de las pruebas sigue un proceso detallado, que se inicia con la elaboración de ítems los cuales, después de una revisión de contenido, son sometidos a procesos de pilotaje con el propósito de enriquecer el banco de ítems para futuras evaluaciones. Los cuadernillos de pruebas que se aplican contienen un total de 35 preguntas, de las cuales treinta forman la parte operativa, es decir, ítems que se utilizan para valorar el rendimiento académico, y cinco preguntas que forman parte del pilotaje.

Si bien es cierto que los informes de rendimiento académico, derivados de las evaluaciones estandarizadas, han brindado información atendiendo a diversas variables de análisis, por ejemplo, nivel de desempeño, porcentaje de respuestas correctas por los diversos componentes de cada bloque, por género, tipo de centro educativo, área geográfica etc. no se han profundizado en analizar detalles propios del comportamiento de cada pregunta en diferentes grupos de análisis, como pueden ser las respuestas que brindan los estudiantes de alto desempeño en las pruebas. 
Es sabido que el aprendizaje de las matemáticas genera dificultades, como lo señala Socas (2007), estas dificultades son de diferente naturaleza, se refuerzan y conectan en redes complejas que se concretan en la práctica como obstáculos y se manifiestan en los alumnos en forma de errores. Afirma, además, que el error debe ser considerado como la presencia, en el alumno, de un esquema cognitivo inadecuado y no sólo la consecuencia de una falta específica de conocimiento o una distracción.

De esta manera, el comportamiento de las respuestas dadas por los estudiantes, al contestar pruebas estandarizadas, es una fuente de información de interés, que nos permite identificar qué dificultades o errores tienen los estudiantes que las contestan.

Desde la didáctica de las matemáticas, los errores que cometen los estudiantes al resolver problemas es parte del proceso, por lo tanto, un elemento de interés es conocerlos, discutir con ellos las posibles causas y presentarles situaciones que les permita modificar sus concepciones erróneas.

Este trabajo de investigación es el primer estudio realizado, tomando como referencia la extensa base de datos generada de las evaluaciones estandarizadas en el contexto hondureño, que busca identificar y analizar las dificultades o errores que cometen los estudiantes de los grados $7^{\circ}, 8^{\circ}$ y $9^{\circ}$ al resolver problemas de aritmética y álgebra.

El estudio se centra en aquellos estudiantes que, de acuerdo con la prueba estandarizada, muestran altos puntajes, nos referimos específicamente al 27\% superior, es decir estudiantes que están por encima del percentil 73.

En el proceso de enseñanza y aprendizaje de las matemáticas, la presencia de errores es un obstáculo para alcanzar las competencias matemáticas necesarias en estos grados, por ello resulta necesario identificarlos para poder plantear alternativas orientadas a superarlos.

Un estudio realizado con profesores que enseñan matemáticas, en el contexto hondureño, revela que el álgebra es uno de los bloques más difíciles de enseñar y que muchos de ellos no tienen los conocimientos, ni didácticos ni de contenido, para enseñarla de manera adecuada en los grados $7^{\circ}$ a $9^{\circ}$ de la educación básica (RAMOS; CASAS, 2017). Consideramos que este trabajo, que muestra el desempeño de los estudiantes, complementa la información dada por los profesores y constituye un insumo para orientar la labor docente.

De acuerdo con lo expuesto anteriormente, en este trabajo buscamos responder a las preguntas:

¿Qué dificultades o errores evidencian los estudiantes de mayor desempeño en matemáticas al resolver problemas algorítmicos o algebraicos? ¿En qué tópicos de las matemáticas se presentan estos errores? 


\section{Marco Teórico}

\subsection{Preguntas, reactivos o ítems de opción múltiple}

Las preguntas de opción múltiple, también conocidas como ítems o reactivos, constituyen una buena herramienta para medir el conocimiento de los estudiantes, y presentan varias ventajas en relación con otros tipos de ítems, algunas de estas ventajas son la confiabilidad y la validez (BRAME, 2013).

La confiabilidad se destaca como una ventaja, debido a que al compararlos, por ejemplo con ítems de verdadero o falso, se reduce la probabilidad de acertar la respuesta correcta por azar, esto los convierte en más confiables, pues entre más opciones tenga el ítem, menos probabilidades de acertar por azar; por otro lado, no presentan problemas con la inconsistencia de jueces que pueden afectar las puntuaciones, ya que son ítems dicotómicos (No existen puntuaciones parciales, solamente correcto o incorrecto).

La validez consiste en qué medida una prueba mide lo que pretende medir, típicamente los estudiantes responden más rápido un ítem de opción múltiple que una pregunta abierta, por lo tanto, las pruebas basadas en ítems de opción múltiple abarcan más contenido de un curso, aumentando, así, la validez de la evaluación (BRAME, 2013).

El Consejo Nacional de Medición en Educación (NCME, por sus siglas en inglés) manifiesta que la validez es el grado en que la evidencia y la teoría respaldan las interpretaciones hechas a partir de los puntajes obtenidos en una prueba. La validez, por lo tanto, es la consideración más importante, en el desarrollo y la evaluación de una prueba (NCME, 2014).

En cuanto a la estructura de los ítems de opción múltiple, estos se forman por un enunciado o base y sus respectivas opciones de respuesta. La base puede estar escrita tanto en forma de pregunta cerrada como en forma de un enunciado o afirmación incompleta. Entre las opciones, solo una pertenece a la respuesta correcta, también es llamada clave, mientras el resto de las opciones o respuestas incorrectas también son llamadas distractores.

Los distractores tienen que cumplir con un requisito de plausibilidad, lo que significa que no deben ser obviamente incorrectos, y los estudiantes que no tengan un dominio claro del contenido puedan considerarlos como posibles respuestas correctas, pero, al mismo tiempo, deben ser fácilmente descartables por los estudiantes que sí, dominan el contenido evaluado en el ítem (BRAME, 2013).

Para muchos expertos como Moreno, Martínez y Muñiz (2006), Haladyna y Rodríguez 
(2013), antes de utilizar la prueba de manera operativa, debe ser aplicada en un estudio piloto para conocer las propiedades psicométricas de los ítems como: dificultad, discriminación, o funcionamiento diferencial, entre otros. El estudio piloto permite, también, identificar la presencia de posibles errores de redacción o inconsistencias relacionadas con el propósito de la prueba.

El pilotaje de ítems es una condición fundamental en las evaluaciones estandarizadas, mediante esta etapa se comprueba si los ítems cumplen la función para la que han sido diseñados.

Según Krishnan (2013), el análisis estadístico o cuantitativo podría ayudar a identificar los ítems problemáticos que pueden haber escapado a la atención de los expertos. Este análisis cuantitativo se realiza después de que la prueba ha sido administrada a los estudiantes, mientras que el análisis cualitativo se realiza previo a la administración, y también posteriormente, sin embargo, ambos comparten un mismo propósito que es evaluar la calidad de los ítems.

\subsection{Aporte de la Teoría Clásica de los Test (TCT)}

En el campo de la evaluación educativa se reconocen dos teorías de análisis de pruebas: La teoría clásica de los test (TCT) y la teoría de respuesta al ítem (TRI). Estas teorías son utilizadas para describir las características de los tests por medio de análisis estadísticos, han sido desarrolladas para que los datos puedan ser interpretados adecuadamente y para facilitar la toma de decisiones en base a información científica (AIKEN, 2003; LEI; WU, 2007).

En el análisis y construcción de pruebas predomina el uso de la Teoría Clásica de los Test. Esta teoría tiene sus raíces en los trabajos pioneros de Spearman, de principios del siglo XX. Bajo esta teoría, el análisis de los reactivos de opción múltiple comúnmente se inicia con cálculos tradicionales que no deben faltar, tales como el índice de dificultad y el índice de discriminación para la respuesta correcta, seguido del análisis del funcionamiento de cada uno de las opciones incorrectas o distractores.

La dificultad se representa por el valor $\mathrm{p}$, es igual al puntaje promedio de todos los estudiantes en el ítem dividido por el puntaje máximo del ítem. Para ítems dicotómicos, donde los posibles puntajes son cero (incorrecto) y uno (correcto), el valor-p corresponde al promedio de los puntajes en el ítem, es decir, es el porcentaje de estudiantes que contestan correctamente el ítem.

Para calcular el índice de discriminación para ítems dicotómicos se utiliza la correlación punto biserial, que es equivalente al coeficiente de correlación de Pearson, que se generaliza 
para ítems dicotómicos y politómicos, se calcula con dos variables que son: las calificaciones en el ítem versus las calificaciones totales en la prueba para cada estudiante.

El rango del coeficiente de correlación de Pearson está en el intervalo [-1,1], un criterio compartido típicamente es que valores mayores a 0.25 , entre la opción correcta y el puntaje total de la prueba, se consideran aceptables para afirmar que un ítem discrimina entre los estudiantes que lograron puntajes mayores en el total de la prueba y los que lograron puntajes menores. Si la correlación es cero, o cercano a cero, el ítem no discrimina, y si es negativa significa que algunos de los estudiantes con mayor desempeño total en la prueba, por alguna razón o razones, han respondido equivocadamente el ítem (AIKEN, 2003).

Un aspecto importante al momento de elaborar ítems de opción múltiple es la selección de los distractores, ya que proporcionan valiosa información sobre cómo los estudiantes responden, permitiendo identificar errores comunes.

En un ítem de opción múltiple, también se puede realizar un análisis gráfico del comportamiento de los distractores y de la respuesta correcta. Este análisis gráfico consiste en representar los porcentajes de estudiantes que han seleccionado cada una de las opciones del ítem, de tal manera que se pueda visualizar fácilmente la calidad del ítem por medio de un gráfico. Esto se logra por medio de la división de los estudiantes en tres grupos, según su desempeño (Bajo, Medio y Alto). El grupo de alto desempeño se define por el $27 \%$ de estudiantes con mayor puntaje en la prueba, y el de bajo desempeño por el $27 \%$ con menor puntaje, mientras que el grupo medio queda conformado por el $46 \%$ restante. Luego se calcula el porcentaje de estudiantes que seleccionó cada opción del ítem para cada uno de los tres grupos establecidos (AIKEN, 2003).

El proceso anterior, es utilizado para analizar el comportamiento de la respuesta correcta y de los distractores en preguntas de opción múltiple, en pruebas de gran escala. De esta manera, se puede identificar posibles errores y/o distractores inapropiados.

\subsection{Dificultades y errores en el aprendizaje de las Matemáticas}

El estudio de las dificultades en el aprendizaje de las matemáticas ha sido una cuestión de permanente interés en Educación Matemática, tanto sus orígenes como sus posibles causas, las cuales, para Socas (1997) se sitúan generalmente en el microsistema educativo: alumno, materia, profesor e institución escolar, y se manifiestan en los alumnos en forma de errores.

Para Rico (1995), señalar los errores puede contribuir positivamente en el proceso de aprendizaje, ya que estos no aparecen por azar, y afirma que todo proceso de instrucción es 
potencialmente generador de errores. Los errores son datos objetivos que encontramos en los procesos de enseñanza y aprendizaje de las matemáticas y forman parte de las producciones de los alumnos durante su aprendizaje.

Una reseña histórica sobre el estudio de los errores en educación es descrita por Del Puerto, Minnaard y Seminara (2006 apud WEINER, 1922). Los autores consideran a Weiner, en Alemania, como el fundador de la investigación didáctica orientada al estudio de errores; en sus investigaciones trató de establecer patrones de errores que explicasen las equivocaciones individuales.

Estudios posteriores, realizados antes de 1960, en Alemania, la Unión Soviética, Estados Unidos y España consistieron, fundamentalmente, en recuentos del número de soluciones incorrectas y en el análisis de los tipos de errores detectados, para poder clasificarlos y, de esta manera, intentar examinar cómo surgen, haciendo inferencias sobre qué factores podrían haberlos provocado (DEL PUERTO; MINNAARD; SEMINARA, 2006).

La revisión bibliográfica sobre dificultades y errores en el aprendizaje de conceptos matemáticos nos lleva a estudiar los obstáculos epistemológicos relacionados, en ese sentido, Brousseau (1997 apud GARROTE; HIDALGO; BLANCO, 2004), define un obstáculo epistemológico como el conocimiento que ha sido en general satisfactorio durante un tiempo para la resolución de ciertos problemas y que, por esta razón, se fija en la mente del estudiante, pero que, posteriormente, resulta inadecuado cuando el alumno se enfrenta a nuevos problemas.

En sus trabajos sobre el aprendizaje de las matemáticas, Brousseau (1997 apud GARROTE; HIDALGO; BLANCO, 2004) distingue diferentes orígenes de esos obstáculos: los vinculados con la metodología, es decir, de origen didáctico y los relacionados con la dificultad intrínseca del concepto, de origen epistemológico.

Al analizar procesos cognitivos en el aprendizaje del álgebra, Palarea y Socas (1999) distinguen entre obstáculo cognitivo y error, al plantear diferentes orígenes de los mismos; de esta manera, definen obstáculos cognitivos como conocimientos que han sido satisfactorios para la resolución de ciertos problemas durante un tiempo, y resultan inadecuados para enfrentarse con otros problemas; errores del álgebra que están en la aritmética cuando el estudiante para entender la generalización de relaciones y procesos se requiere que los problemas sean asimilados en el contexto aritmético y errores del álgebra debidos a características propias del lenguaje algebraico, que son de naturaleza estrictamente algebraico y no tienen referencia explícita en la aritmética.

Si bien la naturaleza de esta investigación no está orientada a identificar las causas de las dificultades y errores que cometen los estudiantes, coincidimos con el planteamiento de 
Socas (1997), al afirmar que éstas pueden asociarse a la propia disciplina, a la complejidad de los objetos de las matemáticas y procesos de pensamiento matemático, a los procesos de enseñanza desarrollados para el aprendizaje, a los procesos de desarrollo cognitivo de los alumnos y, finalmente, pueden estar asociadas a actitudes afectivas y emocionales hacia las matemáticas.

Por otra parte, Radatz (1979) establece cinco categorías generales para clasificar los errores a partir del procesamiento de la información:

1. Errores debidos a dificultades del lenguaje. El aprendizaje de los conceptos, símbolos y vocabulario matemático es, para muchos alumnos, un problema similar al aprendizaje de una lengua extranjera.

2. Errores debidos a dificultades para obtener información espacial, algunas representaciones icónicas de situaciones matemáticas pueden suponer dificultades en el procesamiento de la información.

3. Errores debidos a un aprendizaje deficiente de hechos, destrezas y conceptos previos, se incluyen todas las deficiencias de conocimiento sobre contenidos y procedimientos específicos para la realización de una tarea matemática. Incluye la ignorancia de los algoritmos, conocimiento inadecuado de hechos básicos, procedimientos incorrectos de la aplicación de técnicas y dominio insuficiente de símbolos y conceptos necesarios.

4. Errores debidos a asociaciones incorrectas o a rigidez del pensamiento, la experiencia sobre problemas similares anteriores puede producir una rigidez en el modo habitual de pensamiento y una falta de flexibilidad para codificar y decodificar nueva información.

5. Errores debidos a la aplicación de reglas o estrategias irrelevantes, surgen con frecuencia por aplicar con éxito reglas o estrategias similares en áreas de contenidos diferentes.

Identificar las dificultades y los errores que cometen los estudiantes constituye un verdadero reto en el quehacer docente. Desde nuestro estudio, identificamos los errores tomando de referencia las repuestas incorrectas dadas a preguntas o ítems de opción múltiple.

Resulta importante descubrir las razones que explican el funcionamiento no esperado de un ítem en cuanto a su análisis psicométrico, sin importar el contenido evaluado en el mismo.

En ese sentido, Krishnan (2013) establece que estas razones, generalmente, podrían tener diferentes causas, entre ellas: redacción inadecuada de la pregunta, defectos en el proceso de aprendizaje del contenido evaluado, representar un contenido diferente al que se mide en el resto de la prueba, la presencia de sesgo en un ítem para o contra un subgrupo de la población, la capacidad general del estudiante de comprender el verdadero significado del ítem, 
aumentando las probabilidades de adivinar la respuesta correcta.

\section{Método}

Este estudio sigue una metodología cuantitativa, con orientación descriptiva y exploratoria, mediante el uso y manejo de la base de datos correspondiente a la evaluación nacional fin de grado 2015, en la que se aplicaron pruebas escritas de matemáticas a una muestra nacional de estudiantes hondureños de los grados de $7^{\circ}$ a $9^{\circ}$.

El manejo de la base de datos permitió determinar, con precisión, los elementos de análisis como se detalla a continuación.

\subsection{Elementos de análisis}

Como lo manifestamos anteriormente, el sistema de evaluación implementado en Honduras establece que un porcentaje de las preguntas sean sometidas al proceso de pilotaje, con el propósito de asegurar que las pruebas en las próximas ediciones tendrán ítems de buena calidad psicométrica.

El estudio se realizó mediante el análisis de las respuestas dadas por los estudiantes que contestaron las pruebas de evaluación aplicadas a finales del año académico 2015. En esa evaluación nacional participaron un total de 83,730 estudiantes de los grados $7^{\circ}$ a $9^{\circ}$, cuya edad está entre los doce y quince años, de los dieciocho departamentos del país.

En los grados en estudio se pilotearon un total de 126 ítems (Tabla 1):

Tabla 1 - Cantidad de ítems analizados, por grado

\begin{tabular}{cc}
\hline Grado & Cantidad de ítems de pilotaje \\
\hline $7^{\circ}$ & 37 \\
$8^{\circ}$ & 52 \\
$9^{\circ}$ & 37 \\
Total & 126 \\
\hline
\end{tabular}

\subsection{Identificación de ítems contestados erróneamente por estudiantes de alto desempeño}

De acuerdo con lo establecido en el marco teórico, la selección de los ítems en los cuales los estudiantes de alto desempeño en la prueba se equivocan se realizó siguiendo dos etapas: análisis de correlación de Pearson y análisis gráfico de opciones.

El coeficiente de correlación de Pearson (r), se analiza en dos aspectos, el primero entre la respuesta correcta (clave) de un ítem y el total de la prueba que da la pauta de posibles errores, 
esto es, si $r \leq 0$ significa que estudiantes de alto desempeño en el total de la prueba han seleccionado una respuesta errónea en ese ítem. El segundo es valorar el coeficiente de correlación de cada distractor con el total de la prueba, en este caso, cuando $r \geq 0.15$, en un distractor, significa que estudiantes con alta calificación en la prueba seleccionan una respuesta incorrecta.

Por otra parte, el análisis gráfico de opciones nos permite corroborar los datos obtenidos en la etapa anterior. En este análisis se muestra el porcentaje de estudiantes, según su nivel de desempeño (Bajo, Medio, Alto), que seleccionan cada opción del ítem (A, B, C o D).

Tal y como lo establecimos en la sección 2.2 de nuestro marco teórico, una vez aplicadas las pruebas estandarizadas dividimos los resultados de los estudiantes en tres segmentos, el 27\% de bajo desempeño, el $46 \%$ de desempeño medio y el restante $27 \%$ de alto desempeño.

\section{Presentación, análisis y discusión de resultados}

En los resultados que a continuación presentamos se muestran los ítems, por grado, en los que los estudiantes con buen desempeño en el total de la prueba seleccionan respuestas incorrectas, así mismo, al finalizar los ítems de cada grado se hace su respectivo análisis.

Es claro que la naturaleza de los ítems de opción múltiple no permiten identificar procesos seguidos por los estudiantes para seleccionar cada respuesta, por ello, y a la luz del marco teórico, buscamos analizar las posibles causas.

De acuerdo con el análisis de correlación de los distractores, realizado a los 126 ítems del pilotaje, detectamos un total de catorce ítems relacionados con conceptos algorítmicos o algebraicos en los que los alumnos, que obtienen altas calificaciones en las pruebas fin de grado, seleccionan de forma significativa opciones incorrectas, este detalle se corrobora en el análisis gráfico realizado a cada ítem. Veamos los ítems seleccionados en cada grado.

\section{1 Ítems de $7^{\circ}$ grado}

En este grado encontramos cinco ítems en los que hay evidencias estadísticas de que los estudiantes de alto desempeño en la prueba cometen error; en este grado, cuatro ítems seleccionados se orientan a situaciones algorítmicas propias de la aritmética, como elementos previos al álgebra, y uno relacionado con el concepto de valor absoluto que corresponde a un concepto algebraico, como se muestra a continuación:

Ítem 1: ¿Cuál es el resultado de: $|-3|+|1|$ ? 

A. -4
B. -2
C. 2
D. 4

El ítem 1, relacionado con el concepto de valor absoluto y adición de números enteros fue contestado por 3291 estudiantes, la respuesta correcta es la opción D y los indicadores estadísticos para cada opción se muestran en la Tabla 2.

Tabla 2 - Análisis de opciones ítem 1

\begin{tabular}{ccccc}
\hline & A & B & C & D \\
\hline Porcentaje & $48 \%$ & $30 \%$ & $6 \%$ & $16 \%$ \\
Correlación (r) & -0.07 & $\mathbf{0 . 2 2}$ & -0.05 & -0.14 \\
\hline
\end{tabular}

Fuente: elaboración propia

Debemos destacar que la opción B tiene una correlación positiva de 0.22 y la respuesta correcta (D) tiene correlación negativa de -0.14 que, de acuerdo con nuestro marco teórico, es un indicador de que estudiantes con alta calificación en la prueba están seleccionando erróneamente la opción B.

El análisis gráfico para este ítem, que muestra la Figura 1, muestra claramente que a medida que el nivel de desempeño de los estudiantes aumenta, el porcentaje de estudiantes que seleccionan una opción incorrecta también aumenta.

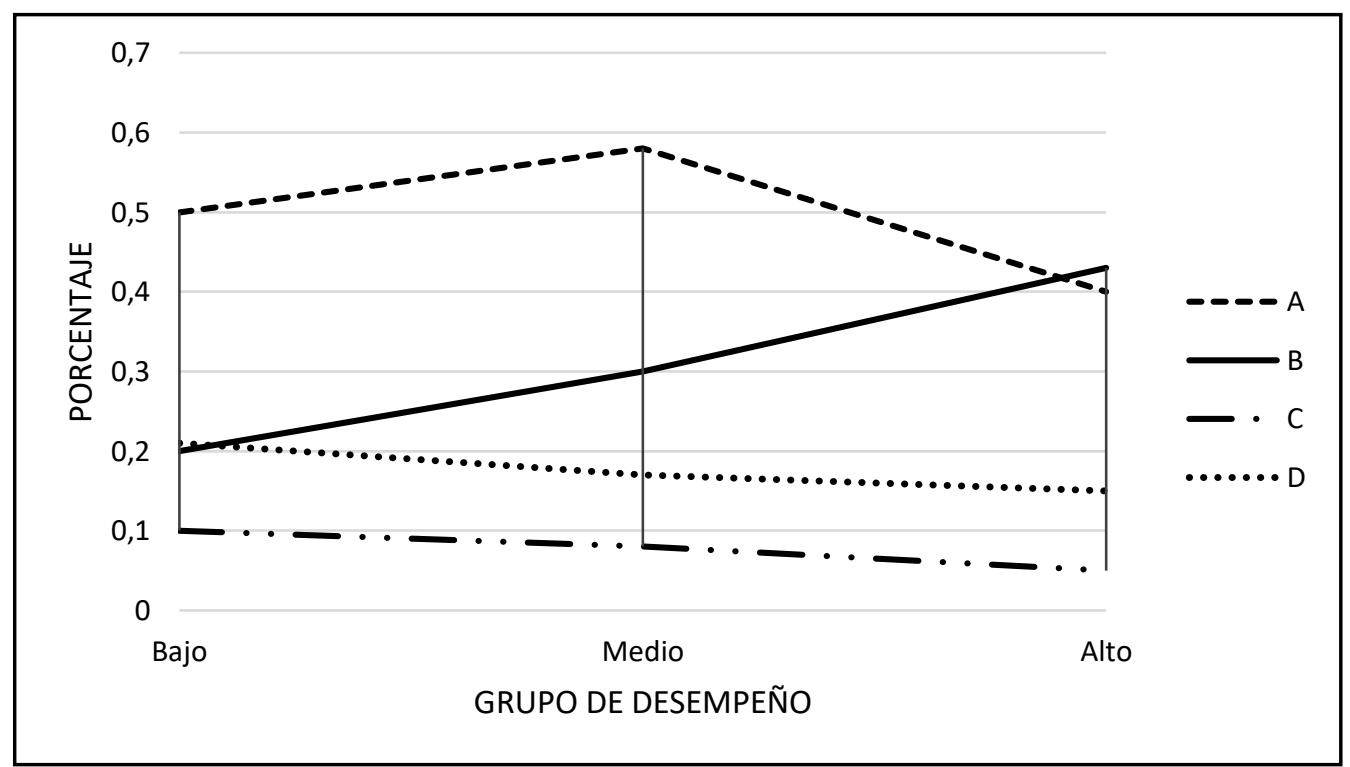

Figura 1 - Análisis gráfico ítem $1,7^{\circ}$ grado Fuente: elaboración propia

Análisis similar efectuamos a los restantes ítems, y estos resultados se muestran en la Tabla 3, que presenta el ítem, la cantidad de estudiantes que lo contestaron (N), la respuesta correcta (Key) y los porcentajes de estudiantes que seleccionaron cada opción con su respectiva correlación $(r)$ : 
Tabla 3 - Ítems selecionados de $7^{\circ}$ grado con sus indicadores

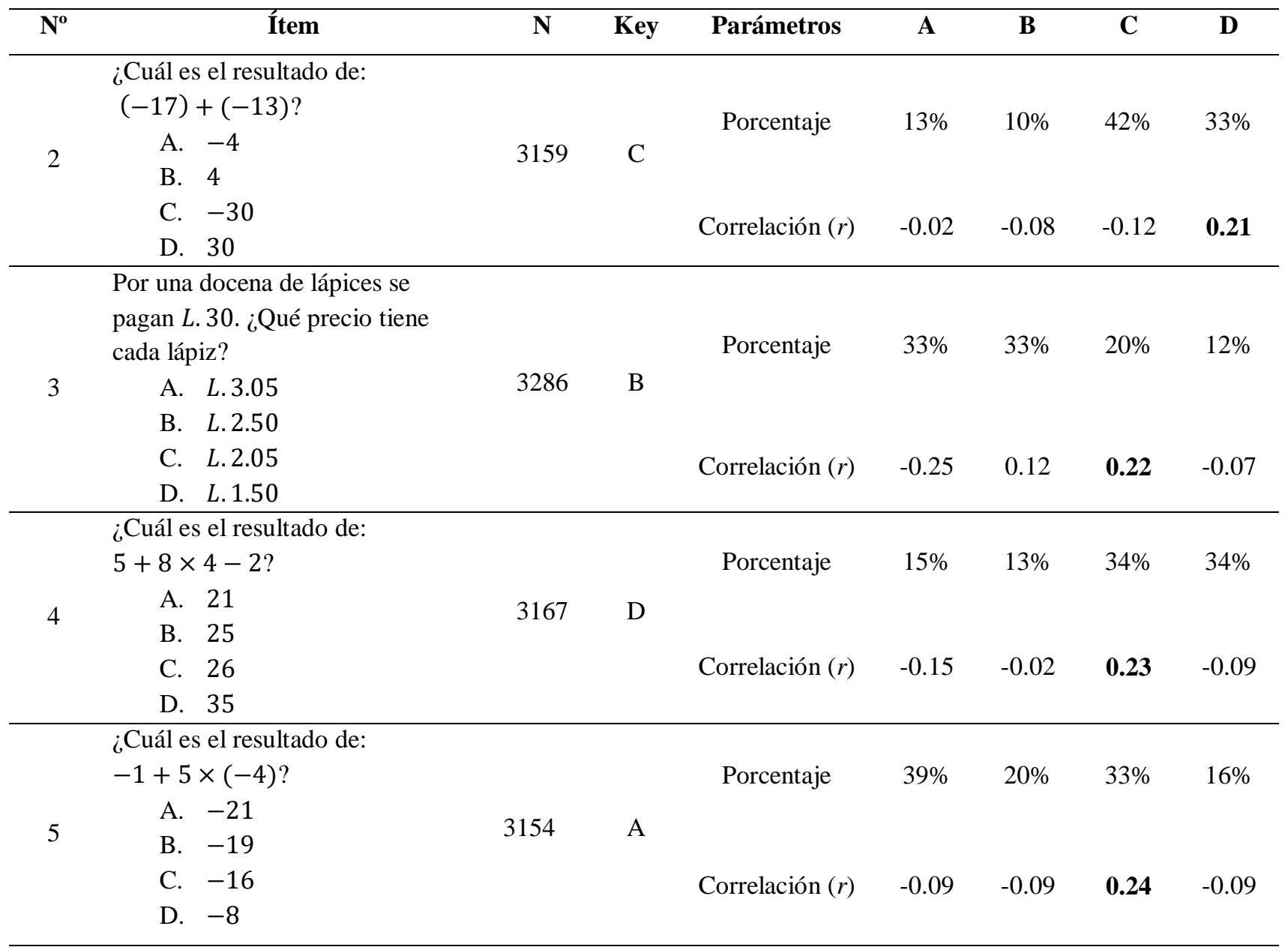

Fuente: elaboración propia

Queda claro que los estudiantes de este grado presentan dificultades en la parte algorítmica de operaciones aritméticas, de acuerdo con lo planteado en nuestro marco teórico esta es la base de errores frecuentes en el álgebra. Se evidencia que en los ítems 4 y 5 más del $30 \%$ de estos estudiantes no conocen la prioridad de las operaciones. Cabe señalar que el error que presentan en el ítem 1 está relacionado directamente con el concepto de valor absoluto, propio del lenguaje algebraico. De acuerdo con Palarea y Socas (1999) los errores relacionados con este concepto se deben a que los estudiantes suelen resolver primero la suma.

\section{2 Ítems de $8^{\circ}$ grado}

En este grado también encontramos seis ítems en los que hay evidencias estadísticas de que los estudiantes de alto desempeño tienen dificultades, como se muestra a continuación:

Ítem 6: ¿Cuál es el resultado de $7 \sqrt{3}-5 \sqrt{3}-2 \sqrt{3}$ ?

A. 0

B. 1 

C. $\sqrt{3}$
D. $-4 \sqrt{3}$

Este ítem relacionado con operaciones básicas con radicales fue contestado por 1942 estudiantes de $8^{\circ}$ grado, tiene como respuesta correcta la opción $\mathrm{A}$, fue respondido correctamente por el $21 \%$ de los estudiantes y tiene una correlación ( $\mathrm{r}$ ) de -0.03 . Los detalles de este ítem se muestran en la Tabla 4:

Tabla 4 - Análisis de opciones ítem 7

\begin{tabular}{ccccc}
\hline & A & B & C & D \\
\hline Porcentaje & $21 \%$ & $9 \%$ & $32 \%$ & $35 \%$ \\
\hline Correlación $(\boldsymbol{r})$ & -0.03 & -0.10 & $\mathbf{0 . 2 0}$ & -0.09 \\
\hline \multicolumn{5}{c}{ Fuente: elaboración propia }
\end{tabular}

Cabe señalar que la opción $\mathrm{C}$ presenta una correlación de 0.20 y ha sido seleccionada por el $32 \%$ de los estudiantes, por lo que se considera que estudiantes de alto desempeño han seleccionado esta opción.

El análisis gráfico de este ítem muestra, claramente, el comportamiento de la opción C, el porcentaje de estudiantes que seleccionan esta opción crece cuando aumenta el nivel de desempeño de los estudiantes.

De igual manera, para $8^{\circ}$ grado se documentaron ítems que muestran respuestas incorrectas por los estudiantes de alto desempeño los cuales se muestran en la Figura 2.

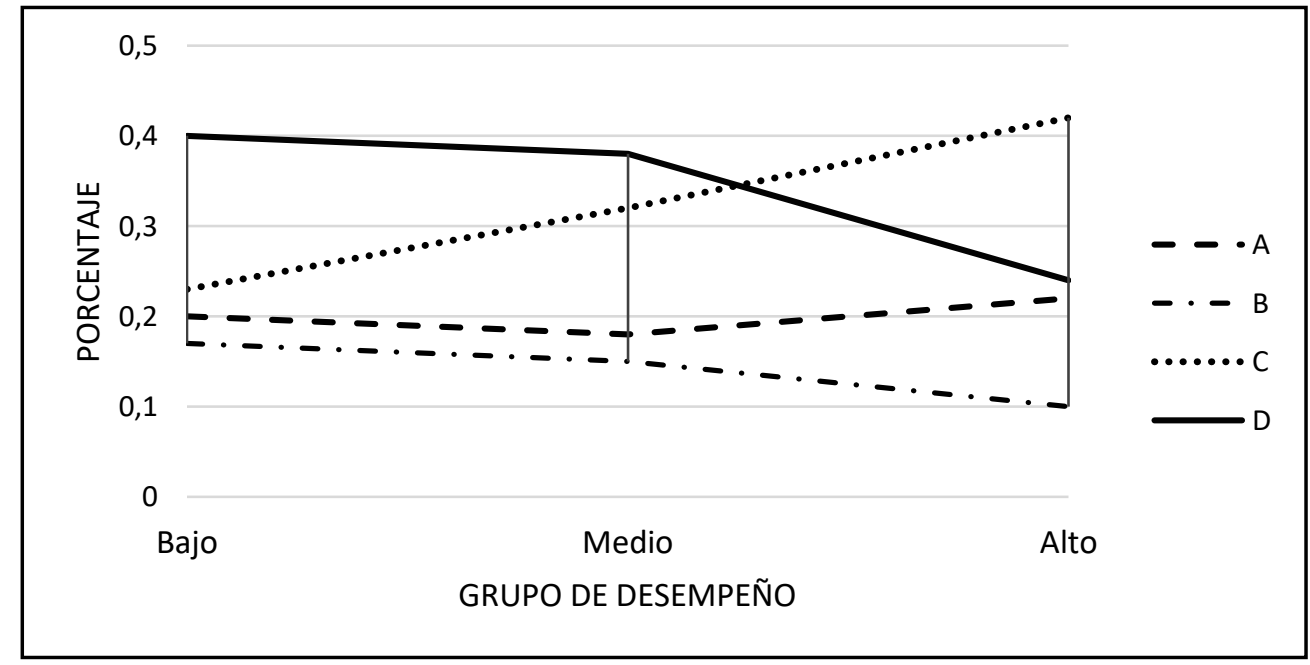

Figura 2 - Análisis gráfico ítem $6,8^{\circ}$ grado Fuente: elaboración propia

Los restantes cinco ítems de este grado tienen las características que se muestran a continuación, en la Tabla 5: 
Tabla 5 - Ítems selecionados de $8^{\circ}$ grado con sus indicadores

\begin{tabular}{|c|c|c|c|c|c|c|c|c|}
\hline $\mathrm{N}^{\mathbf{0}}$ & Ítem & $\mathbf{N}$ & Key & Parámetros & $\mathbf{A}$ & B & $\mathrm{C}$ & D \\
\hline 7 & $\begin{array}{l}\text { ¿Cuál es el resultado de simplificar: } \\
-2 \sqrt{64} \text { ? } \\
\text { A. }-64 \\
\text { B. }-32 \\
\text { C. }-16 \\
\text { D. }-8\end{array}$ & 1858 & $\mathrm{C}$ & $\begin{array}{c}\text { Porcentaje } \\
\text { Correlación (r) }\end{array}$ & -0.17 & -0.03 & 0.04 & 0.17 \\
\hline 8 & 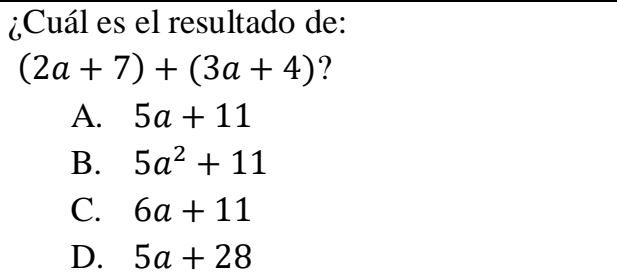 & 1870 & A & Correlación $(r)$ & -0.09 & $29 \%$ & -0.07 & -0.03 \\
\hline 9 & $\begin{array}{l}\text { ¿Cuál es el resultado de: } \\
(5 x+3)^{2} ? \\
\text { A. } 25 x^{2}+9 \\
\text { B. } 25 x^{2}+30 x+9 \\
\text { C. } 25 x^{2}+15 x+9 \\
\text { D. } 10 x^{2}+6\end{array}$ & 11448 & B & Correlación $(r)$ & $51 \%$ & -0.04 & -0.07 & -0.18 \\
\hline 10 & $\begin{array}{l}\text { ¿Cuál es el resultado al simplificar: } \\
\frac{2 x}{x^{2}+2 x} ? \\
\text { A. } \frac{1}{x^{2}} \\
\text { B. } \frac{2}{x+2} \\
\text { C. } \frac{1}{2 x} \\
\text { D. } \frac{x}{x+2}\end{array}$ & 1929 & B & 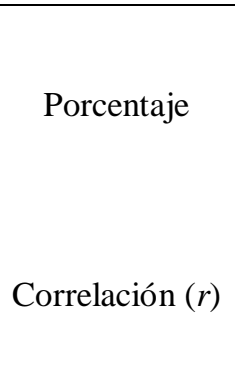 & $22 \%$ & -0.22 & $12 \%$ & $16 \%$ \\
\hline 11 & $\begin{array}{l}\text { ¿Cuál es el resultado de simplificar: } \frac{x^{2}+x}{x} ? \\
\begin{array}{l}\text { A. } x^{2} \\
\text { B. } x^{2}+1 \\
\text { C. } x+1 \\
\text { D. } x^{2}+x\end{array}\end{array}$ & 1975 & $\mathrm{C}$ & Correlación ( $r$ ) & $34 \%$ & -0.10 & $11 \%$ & $29 \%$ \\
\hline
\end{tabular}

Fuente: elaboración propia

En $8^{\circ}$ grado persisten los errores relacionados con aspectos algorítmicos de álgebra y de aritmética, el ítem 6 es el típico problema de no interpretar adecuadamente los coeficientes de términos semejantes, se evidencia que para un $32 \%$ de los estudiantes, la ausencia de un coeficiente es entendido como un cero, es decir, se deduce que para estos estudiantes $0 \sqrt{3}=$ $\sqrt{3}$.

Por otra parte, en el ítem 7 , hay un $25 \%$ de estudiantes que tienen buen desempeño en la prueba que seleccionan la opción $\mathrm{D}$, lo que hace suponer que para ellos $\sqrt{64}=\sqrt{8}=4$ que es común en los estudiantes de secundaria.

El ítem 9, la mayoría de los estudiantes de buen desempeño (51\%) contestan 
erróneamente la opción $A$, este caso es el típico error en el que, como lo plantea Palarea y Socas (1999), se extiende la estructura $(a * b)^{2}=a^{2} * b^{2}$ al caso de la suma $(a+b)^{2}=a^{2}+b^{2}$, de un modo inconsciente, para los alumnos muy natural, a veces incluso después de ser cuestionado.

Los ítems 10 y 11 son evidentes los errores de cancelación de términos ya documentados en los trabajos de Palarea y Socas (1999).

\section{3 Ítems de $9^{\circ}$ grado}

En $9^{\circ}$ se documentaron tres ítems con correlaciones positivas, superiores al $15 \%$, en opciones incorrectas. Estos ítems con sus elementos estadísticos se muestran en la Tabla 6:

Tabla 6 - Ítems seleccionados de $9^{\circ}$ grado con sus indicadores

\begin{tabular}{|c|c|c|c|c|c|c|c|c|}
\hline $\mathbf{N}^{\mathbf{o}}$ & Ítem & $\mathbf{N}$ & Key & Parámetros & $\mathbf{A}$ & $\mathbf{B}$ & $\mathbf{C}$ & D \\
\hline \multirow{5}{*}{12} & $\begin{array}{l}\text { Se inscribe un cuadrado de lado } 10 \mathrm{~cm} \text { en } \\
\text { una circunferencia. ¿Cuál es el radio del } \\
\text { círculo? }\end{array}$ & \multirow{5}{*}{2621} & \multirow{5}{*}{ B } & \multirow[t]{2}{*}{ Porcentaje } & \multirow[t]{2}{*}{$51 \%$} & \multirow[t]{2}{*}{$15 \%$} & \multirow[t]{2}{*}{$15 \%$} & \multirow[t]{2}{*}{$15 \%$} \\
\hline & A. $5 \mathrm{~cm}$ & & & & & & & \\
\hline & B. $5 \sqrt{2} \mathrm{~cm}$ & & & \multirow{3}{*}{ Correlación $(r)$} & \multirow{3}{*}{0.22} & \multirow{3}{*}{-0.17} & \multirow{3}{*}{-0.09} & \multirow{3}{*}{-0.02} \\
\hline & C. $10 \mathrm{~cm}$ & & & & & & & \\
\hline & D. $10 \sqrt{2} \mathrm{~cm}$ & & & & & & & \\
\hline \multirow{5}{*}{13} & ¿Qué número hace verdadera la inecuación & \multirow{5}{*}{2518} & \multirow{5}{*}{ B } & \multirow{4}{*}{ Porcentaje } & \multirow{4}{*}{$28 \%$} & \multirow{4}{*}{$21 \%$} & \multirow{4}{*}{$17 \%$} & \multirow{4}{*}{$31 \%$} \\
\hline & lineal $2 x+1<5 ?$ & & & & & & & \\
\hline & A. 3 & & & & & & & \\
\hline & B. 1 & & & & & & & \\
\hline & C. 4 & & & Correlación $(r)$ & -0.06 & -0.06 & -0.05 & 0.18 \\
\hline \multirow{5}{*}{14} & $\begin{array}{l}\text { D. } \quad \\
\text { Se cobraron L.8 en concepto de merienda a }\end{array}$ & \multirow{5}{*}{10266} & \multirow{5}{*}{$\mathrm{D}$} & \multirow{3}{*}{ Porcentaje } & \multirow{3}{*}{$26 \%$} & \multirow{3}{*}{$44 \%$} & \multirow{3}{*}{$15 \%$} & \multirow{3}{*}{$11 \%$} \\
\hline & $\begin{array}{l}x \text { estudiantes y se recaudó más de L. } 72 . \\
\text { ¿Qué expresión representa la cantidad de } \\
\text { estudiantes que pagaron? }\end{array}$ & & & & & & & \\
\hline & $\begin{array}{l}\text { A. } x=8 \\
\text { B. } x=9\end{array}$ & & & & & & & \\
\hline & C. $x<9$ & & & \multirow[t]{2}{*}{ Correlación $(r)$} & \multirow[t]{2}{*}{-0.08} & \multirow[t]{2}{*}{0.16} & -0.05 & -0.04 \\
\hline & D. $x>9$ & & & & & & & \\
\hline
\end{tabular}

Fuente: elaboración propia

El ítem 12 de este grado, muestra un problema geométrico que se resuelve algebraicamente, esto incrementa su nivel de dificultad dado que no queda claro si el estudiante no conoce los conceptos de figuras inscritas en una circunferencia, el concepto de radio del círculo, la aplicación del teorema de Pitágoras que está implícito, o la solución de una ecuación cuadrática. 
En el caso de los ítems 13 y 14 se manifiesta un error típico, que como lo plantea Garrote, Hidalgo y Blanco (2004), se debe a una deficiente comprensión del concepto de inecuación, muchos no establecen diferencia significativa entre este concepto y el de ecuación.

\section{Conclusiones}

Con este trabajo, hemos documentado una serie de conceptos matemáticos en los cuales estudiantes, que muestran buen desempeño en la prueba estandarizada nacional, tienen dificultades. Reconocemos la importancia de trascender a la identificación de las causas que provocan las dificultades encontradas como un aporte a la educación matemática, sin embargo, estas dificultades, reflejadas en los errores que cometen los estudiantes, deben servir para orientar la práctica de los profesores, la elaboración de recursos educativos de apoyo (libros de texto, guías de estudio y otros) así como en la redacción de ítems para futuras evaluaciones.

Como vemos, se han documentado varios conceptos matemáticos en los que los estudiantes muestran dificultades. Estos conceptos deben abordarse a partir de las estrategias pedagógicas que emplean los docentes, a fin de asegurar que los estudiantes reflexionen sobre los posibles errores y que esto permita enfocarse en conceptos más amplios y puedan darle significado a los objetos matemáticos.

Si consideramos que la información obtenida en este estudio corresponde a resultados de pruebas estandarizadas de matemáticas que se aplican al final del año escolar a escala nacional, queda claro que en $7^{\circ}$ grado las mayores dificultades se presentan en aspectos aritméticos que son fundamentales para entender y dominar los conceptos algebraicos que se abordan con mayor énfasis en los grados de $8^{\circ}$ y $9^{\circ}$. Esto supone un mayor compromiso de parte de los profesores para desarrollar una instrucción más efectiva para lograr los objetivos planteados.

Las dificultades de los estudiantes hondureños relacionadas con el aprendizaje del álgebra y la aritmética, documentados en este trabajo, se clasifican en diferentes tópicos, así en $7^{\circ}$ grado se presentan en los temas relacionados con: valor absoluto y operaciones combinadas con números enteros; en $8^{\circ}$ grado, en simplificación de expresiones racionales algebraicas y de radicales; mientras que, en $9^{\circ}$ grado, en ecuaciones y desigualdades.

Se corrobora uno de los errores clásicos en el aprendizaje del álgebra relacionado con el desarrollo del cuadrado de un binomio, se aprecia que más del $50 \%$ de los estudiantes evaluados de $8^{\circ}$ grado, de alto desempeño, afirman que $(x+b)^{2}=x^{2}+b^{2}$ lo que refleja la 
creencia de un procedimiento incorrecto.

Como lo establecimos en nuestro marco teórico, reconocemos que las dificultades en el aprendizaje de las matemáticas se deben a múltiples situaciones que se relacionan, y que sin duda tienen que ver con el papel del docente, la naturaleza misma de las matemáticas y, particularmente, del álgebra y su simbolismo, así como con actitudes afectivas y emocionales de los alumnos provocadas por el formalismo de las pruebas estandarizadas nacionales.

Queda evidenciado que la información que se ha obtenido de las evaluaciones estandarizadas en matemáticas, en todos los grados de la educación básica $\left(1^{\circ}\right.$ a $\left.9^{\circ}\right)$, puede ser útil para identificar dificultades de los estudiantes en los diferentes bloques de la matemática. Desde el campo de la psicometría se debe impulsar este tipo de investigaciones que van más allá de valorar niveles de desempeño o rendimiento académico.

\section{Referencias}

AIKEN, L. Tests psicológicos y evaluación. México: Pearson Educación, 2003.

BRAME, C. Writing Good Multiple Choice Test Questions. 2013. Disponible en: https://cft.vanderbilt.edu/guides-sub-pages/writing-good-multiple-choice-test-questions/. Acceso: 25 nov. 2020.

DEL PUERTO, S. M.; MINNAARD, C. L.; Y SEMINARA, S. A. Análisis de los errores: una valiosa fuente de información acerca del aprendizaje de las Matemáticas. Revista Iberoamericana de Educación, Madrid, v. 38, n. 4, p. 1-13, 2006.

GARROTE, M.; HIDALGO, J.; BLANCO, L. Dificultades en el aprendizaje de las desigualdades e inecuaciones. SUMA, Badalona, v. 46, p. 37-44, 2004.

HALADYNA, T., RODRÍGUEZ, M. Developing and Validiting Test Items. New York: Routledge, 2013.

KRISHNAN, V. The Early Child Development Instrument (EDI): An item analysis using Classical Test Theory (CTT) on Alberta's data. 2013. Disponible en:

http://www.cup.ualberta.ca/wp/content/uploads/2013/04/ItemAnalysisCTTCUPWebsite_10April13.pd f. Acceso: 20 oct. 2020.

LEI, P. W.; WU, Q. CTTITEM: SAS macro and SPSS syntax for classical item analysis. 2007. Disponible en: https://link.springer.com/content/pdf/10.3758\%2FBF03193021.pdf. Acceso: 12 nov. 2020.

MORENO, R.; MARTÍNEZ, R. J.; MUÑIZ, J. Directrices para la construcción de ítems de elección múltiple. Psicothema, Oviedo, v. 16, n. 3, p. 490-497, 2006.

NCME (National Council on Measurement in Education). Standards for Educational and Psychological Testing. Washington: NCME, 2014.

PALAREA, M. M.; SOCAS, M. M. Procesos cognitivos implicados en el aprendizaje del lenguaje algebraico: Un estudio biográfico. El Guiniguada, Las Palmas de Gran Canaria, n. 8/9, p. 319-336, 1999. 
RADATZ, H. Errors Analysis in Mathematics Education. Journal for Research in Mathematics Education, Reston, v. 9, p.163-172, 1979.

RAMOS, L.; CASAS, L. Demanda Cognitiva de Estándares Educativos y Libros de Texto para la Enseñanza del Álgebra en Honduras. Bolema, Rio Claro (SP), v. 32, n. 62, p. 1134-1151, 2018.

RAMOS, L.; CASAS, L. Enseñanza y evaluación del Álgebra en Honduras: concepciones y prácticas docentes. PARADIGMA Revista de Investigación Educativa. año 24. n. 38, 2018.

RICO, L. Errores y dificultades en el aprendizaje de las Matemáticas. En: KILPATRICK, J.; GÓMEZ, P.; RICO, L. (Eds.). Educación Matemática. Bogotá: Grupo Editorial Iberoamérica, 1995. p. 69-108.

SECRETARÍA DE EDUCACIÓN. Estándares Educativos Nacionales, Español y Matemáticas $\mathbf{1}^{\mathbf{0}}$ a $\mathbf{1 2}^{\mathbf{0}}$ grado. Tegucigalpa: SE Impresiones, 2011.

SECRETARÍA DE EDUCACIÓN. Informe Nacional de Desempeño Académico 2016, Español y Matemáticas, $\mathbf{1}^{\mathbf{0}} \mathbf{a}^{\mathbf{0}}$ grado. Tegucigalpa: SE Impresiones, 2017.

SECRETARÍA DE EDUCACIÓN. Informe Nacional de Desempeño Académico 2017, Español y Matemáticas, $1^{\circ}$ a $9^{\circ}$ grado. Tegucigalpa: SE Impresiones, 2018.

SOCAS, M. Dificultades, obstáculos y errores en el aprendizaje de las Matemáticas en la Educación Secundaria. En: RICO, L. et al. La Educación Matemática en la Enseñanza Secundaria. Barcelona: Horsori, 1997. p. 125-154.

SOCAS, M. Dificultades y errores en el aprendizaje de las Matemáticas: Análisis desde el enfoque Lógico Semiótico. En: Investigación en Educación Matemática, 11., 2007, La Laguna. Actas XI Simposio de la SEIEM, 2007. p. 19-52.

Submetido em 26 de Outubro de 2020. Aprovado em 01 de Março de 2021. 